\title{
Heteronuclear dipolar recoupling of half-integer quadrupole nuclei under fast magic angle spinning
}

\author{
Shin-Jong Huang ${ }^{a, *}$, Shang-Bin Liu ${ }^{b}$, Jerry C.C. Chan ${ }^{a}$ \\ a Department of Chemistry, National Taiwan University, No. 1, Section 4, Roosevelt Road, Taipei 106, Taiwan, ROC \\ ${ }^{\mathrm{b}}$ Institute of Atomic and Molecular Sciences, Academia Sinica, Taipei 106, Taiwan, ROC
}

\section{A R T I C L E I N F O}

\section{Article history:}

Received 15 May 2009

Received in revised form

15 July 2009

Keywords:

Quadrupole nuclei

Heteronuclear dipolar recoupling

Rotary resonance

MAS NMR

\begin{abstract}
A B S T R A C T
An experimental method for the heteronuclear dipolar recoupling of half-integer quadrupole nuclei is proposed. The idea is to manipulate the central transition based on the recoupling technique of spinpolarization-inversion rotary resonance. This method allows the extraction of structural parameters under fast magic-angle spinning. Its validity has been examined by the average Hamiltonian theory and numerical simulations. The initial rotational-echo dephasing arising from the dipolar evolution can be approximated by a parabolic function, from which the heteronuclear van Vleck second moment can be estimated. A factor, estimated from two-spin simulations, is required to account for the effects of the quadrupolar coupling and is rather independent of the geometry and the orders of the spin systems. Our method can facilitate the structural characterization of materials containing half-integer quadrupole nuclei under high-resolution condition. Experimental verification has been carried out on two aluminophosphate systems, namely, $\mathrm{AlPO}_{4}-5$ and $\mathrm{AlPO}_{4}-11$.
\end{abstract}

(c) 2009 Elsevier Inc. All rights reserved.

\section{Introduction}

Magic-angle spinning (MAS) is a standard technique in solidstate NMR spectroscopy because it can provide high-resolution condition by suppressing anisotropic interactions. Nowadays, it is relatively straightforward to reintroduce selected interactions for spin- $\frac{1}{2}$ systems under controlled interference between MAS modulation and spin dynamics manipulated by radiofrequency (rf) pulses [1-8]. The situation becomes more complicated for systems involving quadrupole nuclei $\left(I>\frac{1}{2}\right)$, in which the spin Hamiltonians are usually predominated by the anisotropic quadrupolar interactions. The fact that the rf field $\left(\omega_{1}\right)$ is typically much smaller than the quadrupolar interaction $\left(\omega_{Q}\right)$ hinders the use of conventional recoupling techniques originally developed for spin- $\frac{1}{2}$ systems.

Transfer of population double resonance (TRAPDOR) $[9,10]$ and rotational echo adiabatic passage double resonance (REAPDOR) [11,12] have been proposed for heteronuclear dipolar recoupling involving half-integer quadrupole nuclei based on the population transfer via rotor-synchronized adiabatic passage. Recently, it has been reported that a universal REAPDOR curve can be obtained at the initial dephasing stage for the spin pair system with $I=\frac{1}{2}$ and $S=\frac{5}{2}$ [13]. Nevertheless, it is not always possible to achieve the socalled adiabatic passage under the conditions of very fast MAS or

\footnotetext{
* Corresponding author. Fax: +886223636359.

E-mail address: shingjonghuang@ntu.edu.tw (S.-J. Huang).
}

weak rf field. Over the past decade, the rotary resonance recoupling $\left(R^{3}\right)$ technique, originally developed for spin $-\frac{1}{2}$ systems $[3,6]$, has been applied for half-integer quadrupole nuclei in the central-transition subspace [14-16]. The $\mathrm{R}^{3}$ technique has the advantage that the rf field required is comparable to the MAS frequency. In principle, the $\mathrm{R}^{3}$ can be applied for the recoupling of various anisotropic interactions such as homo- or hetero-nuclear dipole-dipole interactions [15]. Consequently, following the same idea significant efforts have been devoted to develop homonuclear dipolar recoupling methods for dipolar correlations of half-integer quadrupole nuclei [17], such as $R^{3}$ [18-20], finite-pulse radio frequency driven (fpRFDR) [21], and symmetry based pulse sequences [22]. On the other hand, considerable progress in heteronuclear dipolar recoupling involving quadrupole nuclei has also been made. In general, the recoupling pulses are applied on the spin- $\frac{1}{2}$ nuclei [23-29] mainly because rf irradiation on quadrupole nuclei may invoke complicated spin dynamics [17]. Nevertheless, the development of the heteronuclear dipolar recoupling techniques for quadrupole nuclei is still an important and challenging topic, which may find potential applications particularly for systems involving two different half-integer quadrupole nuclei.

This work aims to develop a heteronuclear dipolar recoupling scheme for quantitative extraction of structural information in materials containing half-integer quadrupolar nuclei under fast MAS. Compared to the $R^{3}$, the technique of spin polarization inversion rotary resonance recoupling $\left(\mathrm{SPI}-\mathrm{R}^{3}\right)$ is found to be a more robust sequence for heteronuclear dipolar recoupling. Based 
on the average Hamiltonian theory [15], the initial dephasing of the rotational echo arising from the heteronuclear dipolar recoupling of the SPI- $\mathrm{R}^{3}$ can be described by a parabolic function of the evolution time, from which the corresponding van Vleck second moment $\left(M_{2}\right)$ can be extracted. The effects of geometries, orders, and multiple sites in multispin systems are investigated by numerical simulations. Our approach is validated by experimental results obtained for $\mathrm{AlPO}_{4}-5$ and $\mathrm{AlPO}_{4}-11$.

\section{Theory}

Consider an isolated dipolar-coupled spin pair consisting of a spin $-\frac{1}{2}$ nucleus $(S)$ and a half-integer quadrupole nucleus $(I)$. The total Hamiltonian of the I nucleus in the high-field approximation can be written as

$H_{I}(t)=H_{Q}^{(1)}(t)+H_{Q}^{(2)}(t)+H_{C S}(t)+H_{D}(t)+H_{r f}(t)$,

where $H_{Q}^{(1)}(t)$ and $H_{Q}^{(2)}(t)$ represent the first-order and secondorder quadrupolar interactions, respectively, whereas $H_{C S}(t)$ and $H_{D}(t)$ denote contributions from the chemical shift and heteronuclear dipole-dipole interactions, respectively. For simplicity, the homonuclear dipole-dipole interaction is ignored. The term $H_{r f}(t)$, which represents the rf Hamiltonian, can be expressed as $\omega_{r f} I_{x}$ in the rotating frame. According to the works by Baldus et al. [14] and Wi et al. [15], the Hamiltonians of the satellite transitions and multiple-quantum transitions may be ignored in the sudden regime $\left(\omega_{1}^{2} / \omega_{r} \omega_{Q} \ll 1\right)$. Under the continuous rf irradiation in the sudden regime, the effective Hamiltonian for the central transition in the fictitious operator spin- $\frac{1}{2}$ formalism can be written as [15] $\tilde{\tilde{H}}_{I}^{C T, t}(t)=-\left(\Omega_{C S}^{C T}(t)+\Omega_{D}^{C T}(t) S_{z}\right.$

$$
\left.+\Omega_{Q}^{C T}(t)\right)\left(I_{+}^{C T} e^{-i(I+(1 / 2)) \omega_{r f} t}+I_{-}^{C T} e^{i(I+(1 / 2)) \omega_{r f} t}\right),
$$

where $C T$ represents the central-transition subspace. At the rotary resonance condition, i.e. $\omega_{r f}=2 n \omega_{r} /(2 I+1)$, where $n=1$ or 2 , the terms associated with chemical shift anisotropy (CSA), heteronuclear dipolar interaction, and the second-order quadrupolar interactions will be recoupled. To the lowest order, the average Hamiltonian over a rotor period is [15]

$\tilde{\tilde{H}}_{I, R^{3}}^{C T, t}=-\sum\left(\Omega_{ \pm, C S, R^{3}}^{C T}+\Omega_{ \pm, D, R^{3}}^{C T} S_{z}+\Omega_{ \pm, Q, R^{3}}^{C T} I_{ \pm}^{C T}\right.$.

For the dipolar term, we have

$\Omega_{ \pm, D, R^{3}}^{C T}=\omega_{\bar{D}}^{ \pm n}\left(\beta^{D}\right)$,

where for $n=1$ or 2

$\omega_{D}^{ \pm 1}\left(\beta^{D}\right)=\frac{d_{I S}}{\sqrt{2}} m_{I} \sin \left(2 \beta^{D}\right) \exp \left( \pm i \gamma^{D}\right)$,

$\omega_{D}^{ \pm 2}\left(\beta^{D}\right)=\frac{-d_{I S}}{2} m_{I} \sin ^{2}\left(\beta^{D}\right) \exp \left( \pm 2 i \gamma^{D}\right)$

and

$d_{I S}=2 \pi D_{I S}=-\frac{\mu_{0}}{4 \pi} \frac{\gamma_{I} \gamma_{S} \hbar}{r_{3}}$.

Eq. (4) is identical to the results expected for spin- $\frac{1}{2}$ systems. However, the validity of Eq. (4) would rely on the following condition:

$H_{Q}^{(1)} \gg\left(I+\frac{1}{2}\right) \omega_{r f}>\Omega_{C S}^{C T}, \Omega_{Q}^{C T}, \Omega_{D}^{C T}$.

By the same token, the average Hamiltonian for the first rotor period in SPI- $\mathrm{R}^{3}$ is identical to that for the $\mathrm{R}^{3}$ recoupling,

$\tilde{\tilde{H}}_{I, S P I(1)}^{C T, t}=-\sum\left(\Omega_{ \pm, C S, R^{3}}^{C T}+\Omega_{ \pm, D, R^{3}}^{C T} S_{z}+\Omega_{ \pm, Q, R^{3}}^{C T}\right) I_{ \pm}^{C T}$, whereas the average Hamiltonian for the second rotor period, in which the phase of the rf field is inverted, can be derived by inverting the sign of $\omega_{r f}$ in Eq. (2):

$\tilde{\tilde{H}}_{I, S P I(2)}^{C T, t}=-\sum\left(\Omega_{\mp, C S, R^{3}}^{C T}+\Omega_{\mp, D, R^{3}}^{C T} S_{z}+\Omega_{\mp, Q, R^{3}}^{C T}\right) I_{ \pm}^{C T}$.

The lowest-order average Hamiltonian for the entire SPI- $\mathrm{R}^{3}$ sequence can be obtained by taking the average over two successive rotor periods [30]:

$\tilde{\tilde{H}}_{I, S P I-R^{3}}^{C T, t}=-\left(\Omega_{C S, S P I-R^{3}}^{C T}+\Omega_{D, S P I-R^{3}}^{C T} S_{z}+\Omega_{Q, S P I-R^{3}}^{C T}\right) I_{z}^{C T}$,

where, for the dipolar term,

$\Omega_{D, S P I-R^{3}}^{C T}=\frac{\omega_{D}^{+n}\left(\beta^{D}\right)+\omega_{D}^{-n}\left(\beta^{D}\right)}{2}$.

On the basis of Eqs. (4) and (12), the recoupling efficiency of $\mathrm{R}^{3}$ irradiation seems to be higher than that of SPI- $R^{3}$. However, since the terms in Eq. (3) do not commute with each other, the recoupling efficiency of the dipolar term will be sensitive to the tensor orientations and the principal tensor components of the chemical shift and the second-order quadrupolar interaction:

$\left[\Omega_{ \pm, D, R^{3}}^{C T} S_{Z} I_{ \pm}^{C T}, \Omega_{ \pm, C S, R^{3}}^{C T} I_{ \pm}^{C T}\right] \neq 0,\left[\Omega_{ \pm, D, R^{3}}^{C T} S_{Z} I_{ \pm}^{C T}, \Omega_{ \pm, Q, R^{3}}^{C T} I_{ \pm}^{C T}\right] \neq 0$.

On the other hand, all the terms in Eq. (11) commute with one another. Thus, the dipolar recoupling by SPI- $\mathrm{R}^{3}$ will not be affected by other recoupled interactions if the higher order effects are negligible.

For an isolated spin-pair system consisting of a spin- $\frac{1}{2}$ nucleus $(S)$ and a half-integer quadrupole nucleus $(I)$, the rotational echo of the $S$ spins will be attenuated by the $I$-spin irradiation under the condition of $\mathrm{R}^{3}$ or SPI- $\mathrm{R}^{3}$. For a powder sample, one obtains

$\frac{\Delta S}{S_{0}}=\frac{2}{2 I+1}\left(1-\frac{1}{4 \pi} \int_{0}^{2 \pi} d \alpha^{D} \int_{0}^{\pi} \sin \beta^{D} \cos (\Delta \Phi) d \beta^{D}\right)$

where $\Delta \Phi$ is the dephasing angle arising from the dipolar recoupling. According to Eqs. (4) and (12), the dephasing angle for $\mathrm{R}^{3}$ with $n=1$ can be deduced as

$\Delta \Phi_{R^{3}}=\frac{d_{I S} \sin \left(2 \beta^{D}\right)}{2 \sqrt{2}} N T_{r}$,

and for SPI- $\mathrm{R}^{3}$ recoupling with $n=1$ as

$\Delta \Phi_{S P I-R^{3}}=\frac{d_{I S} \sin \left(2 \beta^{D}\right) \cos \gamma^{D}}{2 \sqrt{2}} N T_{r}$,

where $T_{r}$ is the rotor period and $N$ is the number of the rotor cycle throughout the recoupling irradiation. The factor of $2 /(2 I+1)$ accounts for the population of the spin states of $m= \pm \frac{1}{2}$. Although Eqs. (14)-(16) do not have any explicit dependence on the nuclear quadrupole coupling constant $\left(C_{Q}\right)$, if the second-order quadrupolar effect is so large that Eq. (8) becomes invalid, the dipolar recoupling efficiency will be affected by the magnitude of $C_{Q}$ (see below).

\section{Experimental method}

All solid-state NMR experiments were performed on a widebore Bruker Avance 300 spectrometer equipped with a $4 \mathrm{~mm}$ triple resonance MAS probe with a sample spinning rate of $10 \mathrm{kHz}$. The Larmor frequencies for ${ }^{27} \mathrm{Al}$ and ${ }^{31} \mathrm{P}$ were 78.21 and $121.51 \mathrm{MHz}$, respectively. The rf field strengths for the ${ }^{27} \mathrm{Al}$ and ${ }^{31} \mathrm{P}$ channels were 3.33 and $50 \mathrm{kHz}$, respectively. The pulse sequences used were shown in Figs. 1a and b. Saturation combs were applied before the relaxation delays for all experiments. The relaxation delay was $8 \mathrm{~s}$. A total of 64 transients were 


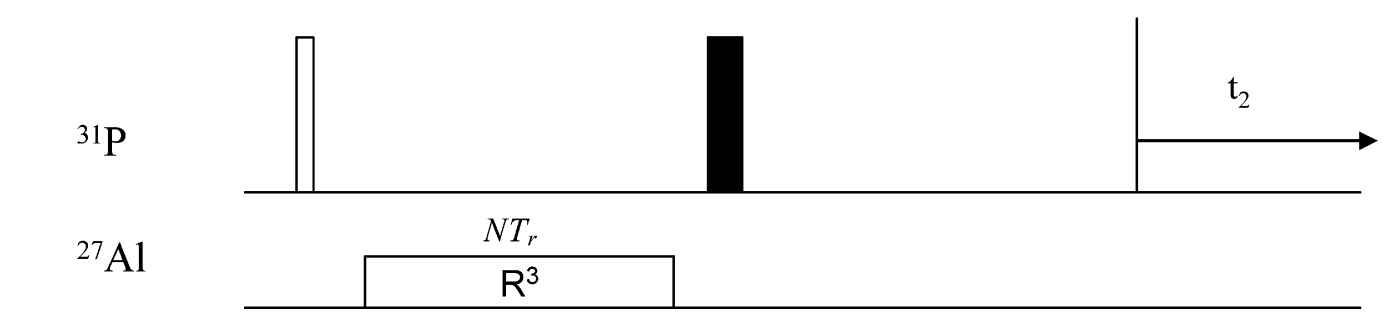

Rotor period

$\begin{array}{llllllll}1 & 1 & 1 & 1 & 1 & 1 & 1\end{array}$

b

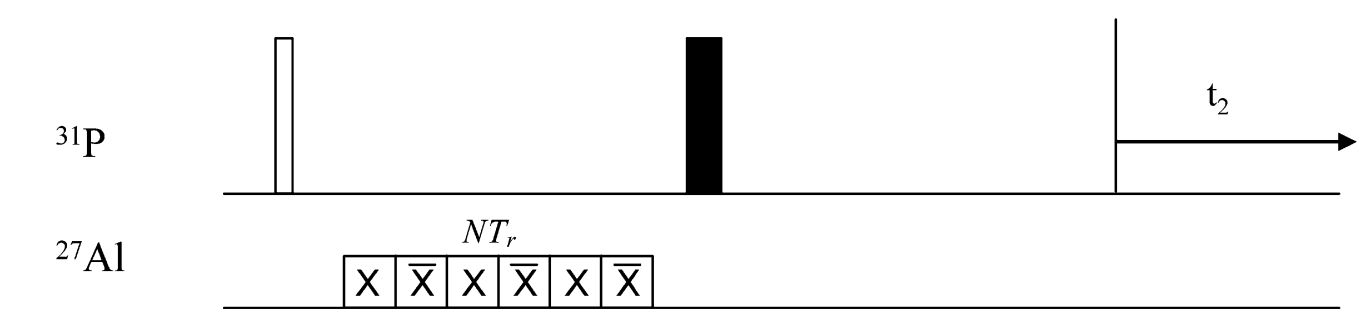

Rotor period

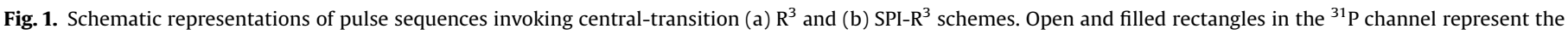

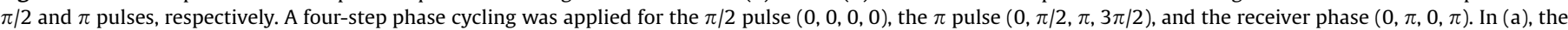

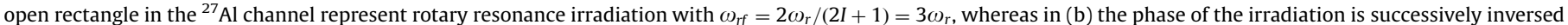
for each rotor period.

accumulated for each spectrum. Two model compounds, namely anhydrous $\mathrm{AlPO}_{4}-5$ and $\mathrm{AlPO}_{4}-11$ powders, were first dehydrated under vacuum at $473 \mathrm{~K}$ for $24 \mathrm{~h}$ and sealed in a gas-tight $\mathrm{ZrO}_{2}$ rotor under $\mathrm{N}_{2}$ atmosphere. To ensure a good rf homogeneity, the sample was packed in the middle one-third of the rotor volume by Teflon spacers.

All simulations were carried out on SIMPSON package (version 1.1.2) [31]. The maximum time step $(\max d t)$ was set to be $0.1 \mu \mathrm{s}$. A powder-averaging scheme containing 320 REPULSION angles (alpha and beta) and 18 gamma angles was chosen [32]. Simulations were done based on the pulse sequences shown in Figs. 1a and b. The parameters such as rf field strength and spinning frequency were matched to the experimental conditions. The real part of the calculated FIDs was taken for analyses, whereas the negligible amplitude of the imaginary part was ignored. Relaxation effects were not taken into account. Data convergence was checked by repeating selected calculations with smaller time steps and more extensive powder-averaging schemes.

\section{Results and discussion}

\subsection{Isolated dipolar-coupled spin pair system}

Two-spin $\left(S={ }^{31} \mathrm{P}\right.$ and $\left.I={ }^{27} \mathrm{Al}\right)$ simulations of ${ }^{31} \mathrm{P}$ echo dephasing upon heteronuclear dipolar evolution time were carried out based on $\mathrm{R}^{3}$ (Fig. 1a). Fig. 2a shows the simulated dephasing curves with respect to the dimensionless parameter $\left|N T_{r} D_{I S}\right|$ for a series of $C_{Q}$, ranging from 2 to $8 \mathrm{MHz}$. Two sets of simulations were obtained for $D_{I S}$ equal to -400 and $-800 \mathrm{~Hz}$. The asymmetry parameter of the quadrupolar interaction $(\eta)$ was set to be zero and the z-axis of the principal axis system (PAS) of the dipolar tensor was set to be coaxially aligned with that of the electric field gradient tensor. The dephasing curves calculated by the SIMPSON package are shown along with the dephasing curve predicted by the average Hamiltonian theory (Eqs. (14) and (15)) with $n=1$, as indicated by the dashed line. The initial parts of the dephasing curves are well approximated by Eqs. (14) and (15), for which the $C_{Q}$ seems to have minor effect. However, the dephasing curves were found to vary strongly with the relative orientation between the PAS coordinates of the heteronuclear dipolar and the electric field gradient tensors (Fig. 2b), and the asymmetry parameter $\eta$ (Fig. 2c). These strong dependences can be ascribed to the fact that the recoupled dipolar and second-order quadrupolar interactions do not commute as revealed in Eq. (13). The interference effect among these recoupled interactions reduces the efficiency of the dipolar dephasing. It should be pointed out that in most systems of interest the relative orientations between the PAS coordinates of anisotropic interactions are unknown and cannot be determined easily. Therefore, such dependence on the relative orientations between the PAS coordinates of anisotropic interactions would severely limit the applicability of $\mathrm{R}^{3}$ to dipolar recoupling for halfinteger quadrupole systems.

In the case of SPI- ${ }^{3}$ (Fig. 1b), the dephasing curves were calculated with respect to the $C_{Q}$ values, the tensor orientations, and the asymmetry parameter $\eta$ (Figs. 3a-c). Unlike the case of $\mathrm{R}^{3}$ recoupling, the lowest-order average Hamiltonians for SPI- $\mathrm{R}^{3}$ do commute with itself at different times. The dephasing curves become rather independent of the tensor orientation and $\eta$ at the short evolution regime, as shown in Figs. $3 \mathrm{~b}$ and c, respectively. However, we observe that the initial dephasing of the rotation echo arising from SPI- $R^{3}$ depends critically on $C_{Q}$ (Fig. 3a). We do not fully understand this phenomenon. As mentioned above, the validity of Eq. (8) mainly depends on two conditions: (i) The rf field should be sufficiently small so that the satellite transitions would not affect the spin dynamics significantly and (ii) the rf field should be larger than the second-order quadrupolar interaction. Apparently, this undesirable dependence on $C_{Q}$ is originated from the second-order quadrupolar interaction. We have carried out additional simulations in which the second-order 
b

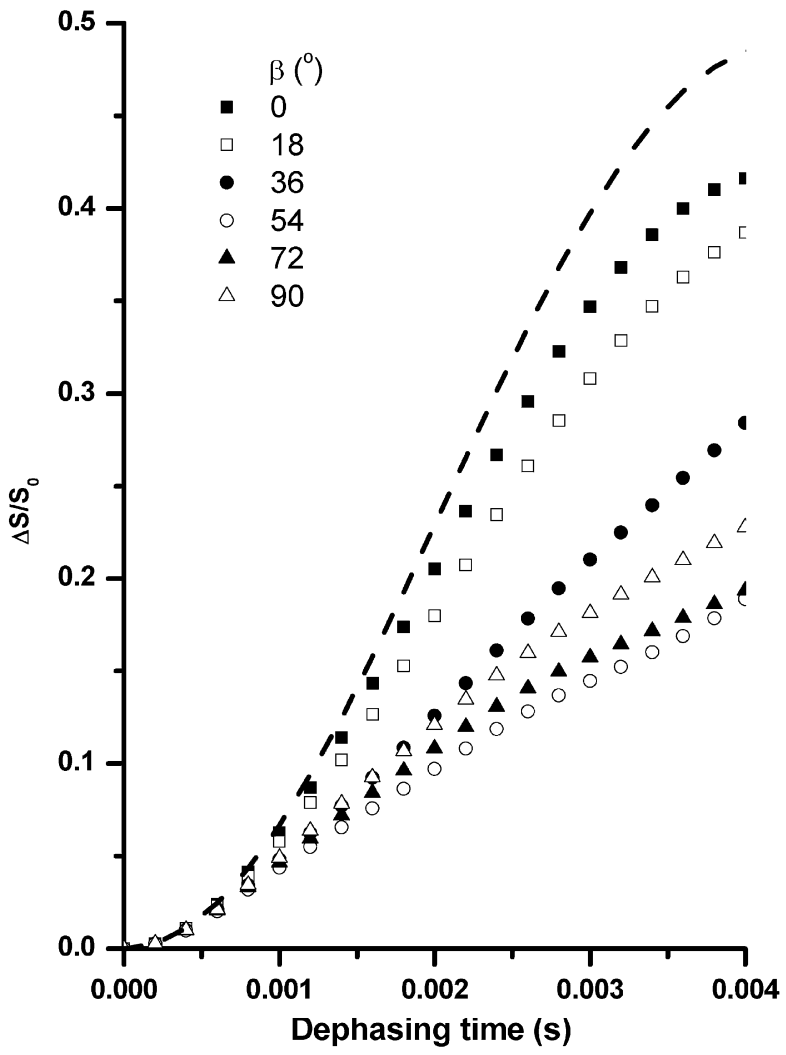

a

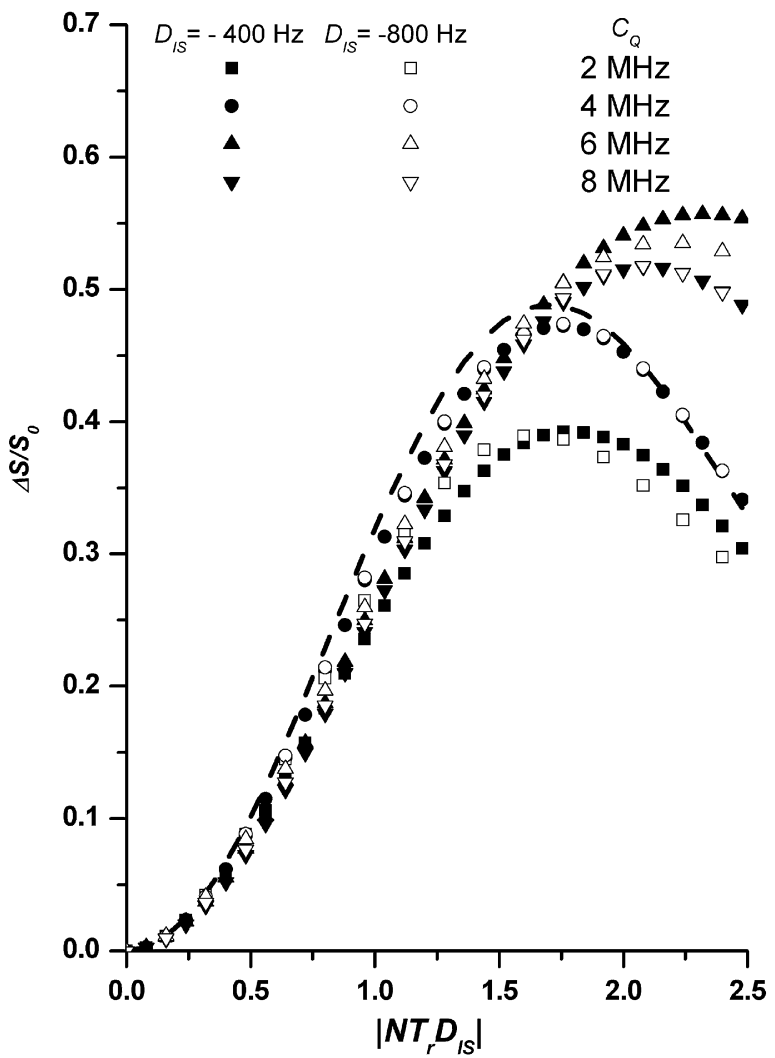

$\mathrm{C}$

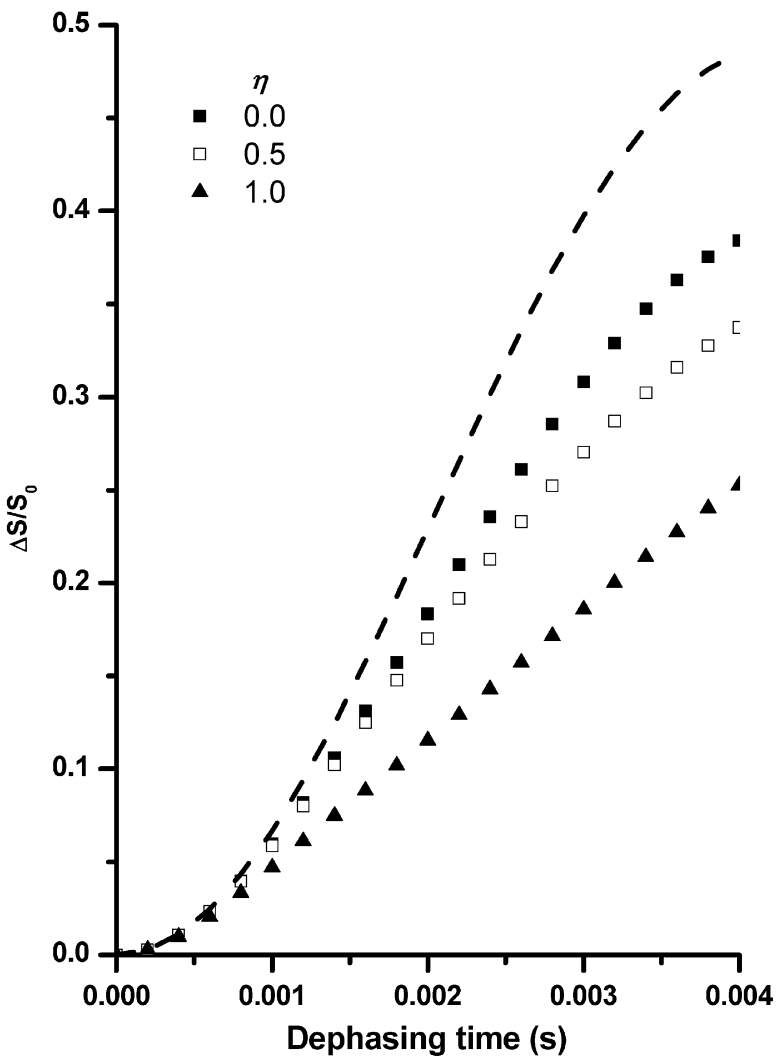

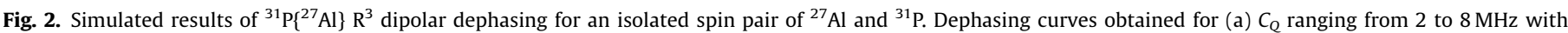

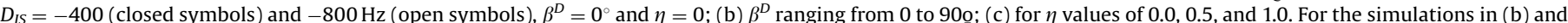
(c), we have $C_{Q}=2.5 \mathrm{MHz}, \eta=0, D_{I S}=-400 \mathrm{~Hz}$, and $\alpha^{Q}=\beta^{Q}=\gamma^{Q}=\alpha^{D}=\gamma^{D}=0 \underline{0}$. The dashed line represents the curve calculated by Eqs. (14) and (16). 
a

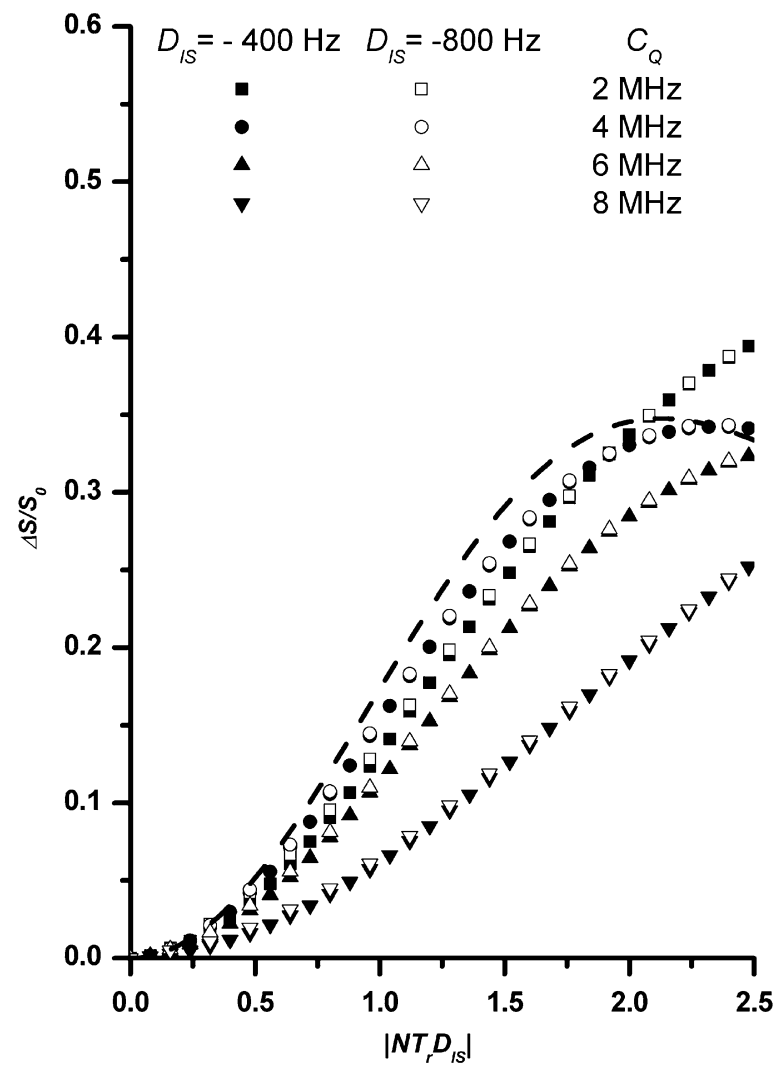

C

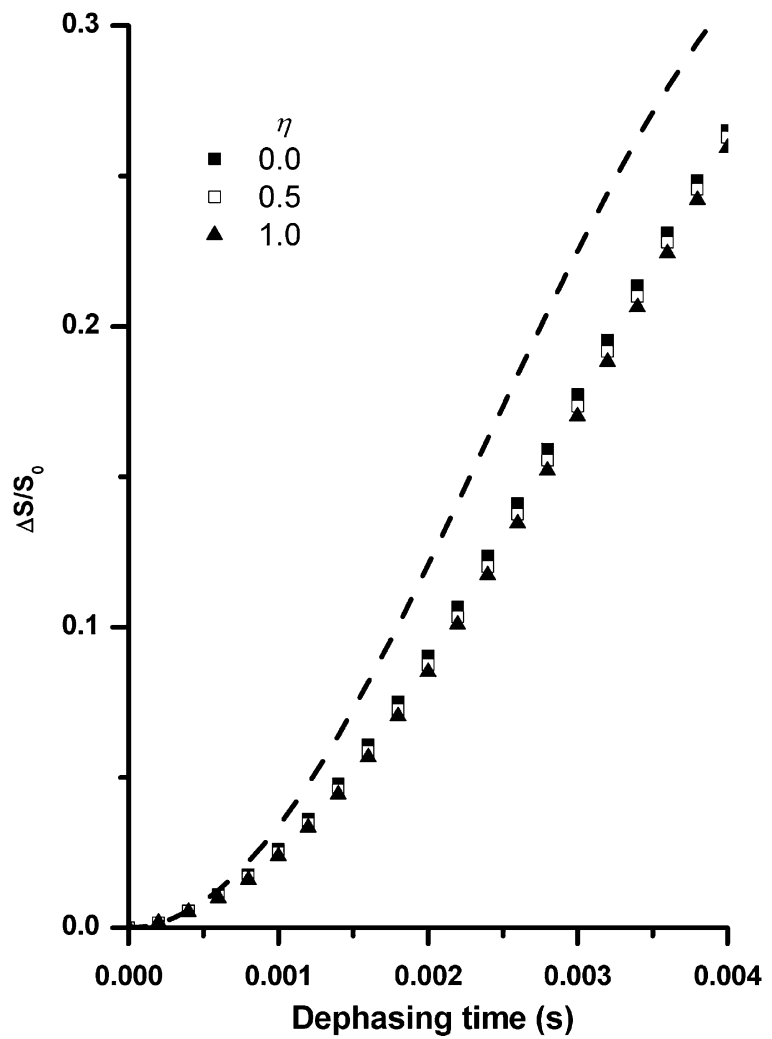

b

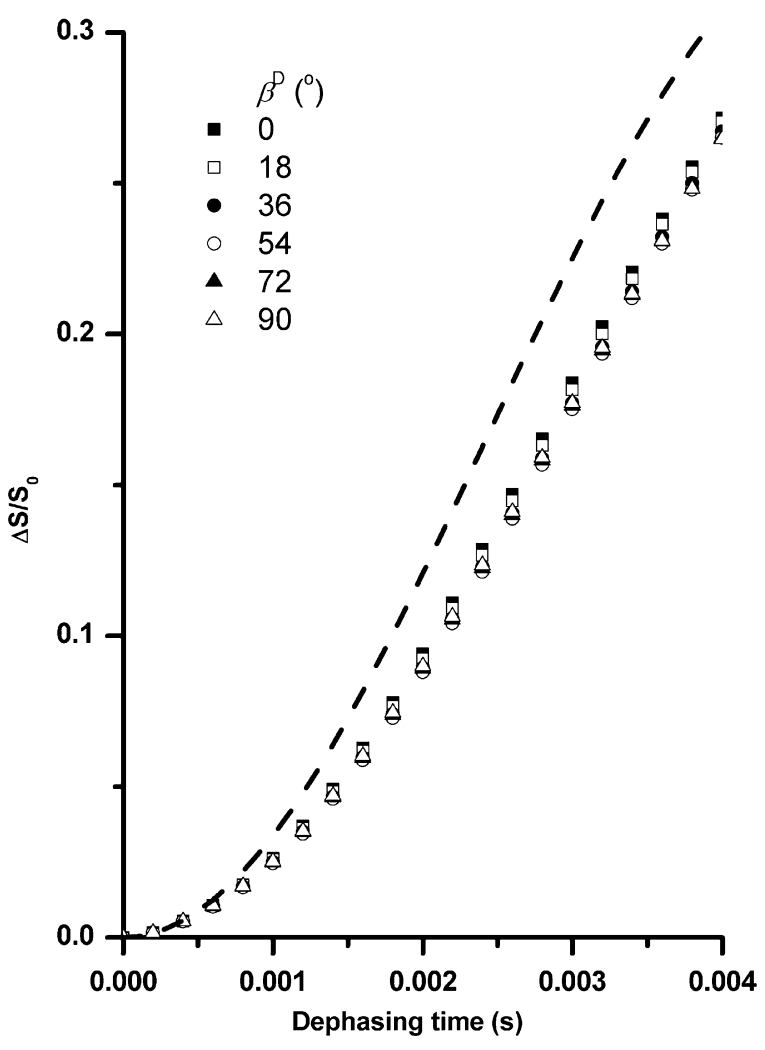

d

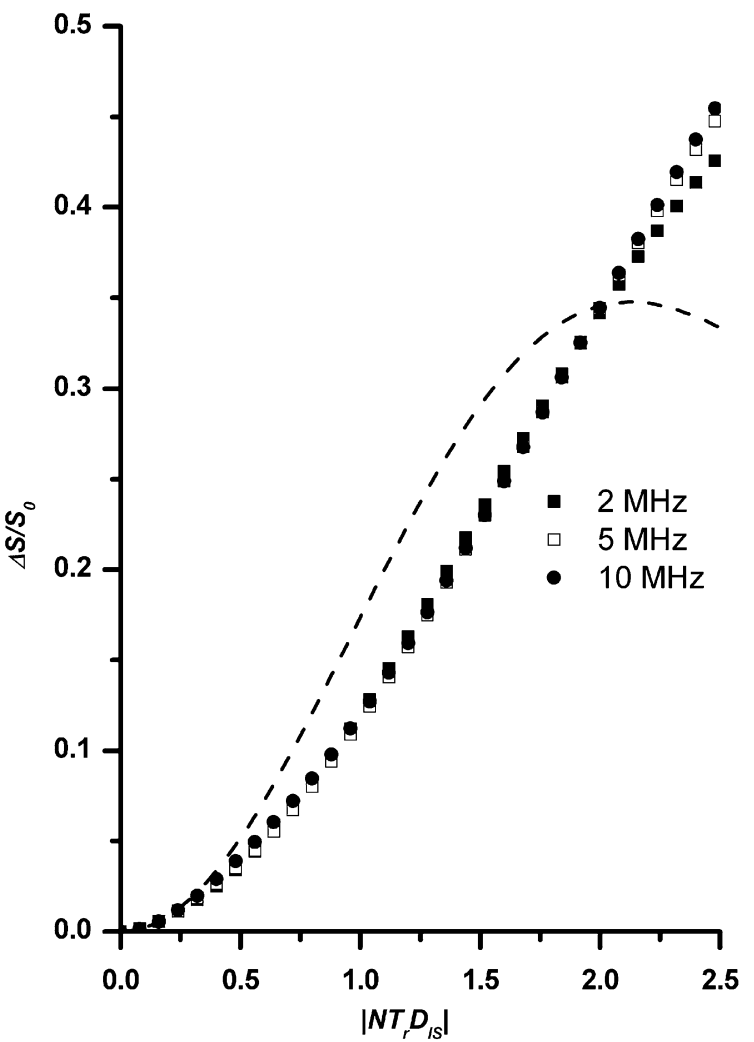

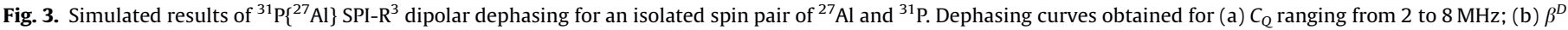

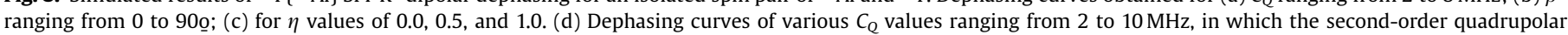
interaction is intentionally switched off. Other simulation parameters are referred to the caption of Fig. 2. 
quadrupolar interaction is intentionally omitted. Indeed, the behavior of the dipolar dephasing becomes less dependent on $C_{Q}$ value (Fig. $3 \mathrm{~d}$ ).

Although the dipolar dephasing of SPI- $\mathrm{R}^{3}$ is dependent on $C_{Q}$, we will demonstrate in the next section that a scaling factor can be used to account for such dependence. Once the amplitude of the scaling factor is evaluated by SIMPSON simulations (vide infra), SPI- $\mathrm{R}^{3}$ may be applicable for the determination of $D_{I S}$ in a manner similar to that proposed for REDOR analyses involving half-integer quadrupole nuclei [41-43].

\subsection{Parabolic approximation for the initial signal attenuation}

It has been reported that the initial REDOR dephasing curve is geometry independent and hence allows for extraction of the van Vleck second moment $\left(M_{2}\right)$ for multiple-spin systems [44-46]. Such approach has also been successfully extended to systems involving quadrupole nuclei $[42,43]$. Following the same idea, an analytical formula was deduced to describe the initial part of the dephasing curve for SPI- ${ }^{3}$ on the basis of Eqs. (14) and (16):

$\left(\frac{\Delta S}{S_{0}}\right)_{S P I-R^{3}}=\frac{\pi^{2}}{15} \frac{2}{2 I+1} f\left(N T_{r} D_{I S}\right)^{2}$

where the scaling factor $f$ is introduced herein to account for the influence of $C_{Q}$ on the echo attenuation. Referring to Figs. 3a-c, all dephasing curves can be approximated by parabolic functions at short dephasing times. Accordingly, the factor $f$ for a specific $C_{Q}$ value may readily be deduced by fitting the simulated curves in the initial regime to Eq. (17). In other words, once the $C_{Q}$ value for a system of interest is determined by experiments such as MQMAS [33-36] and STMAS [37-40], $f$ can be estimated through SIMPSON two-spin simulations. For multispin systems, Eq. (17) may be reformulated as

$$
\begin{aligned}
\left(\frac{\Delta S}{S_{0}}\right)_{S P I-R^{3}} & =\frac{\pi^{2}}{15} \frac{2}{2 I+1} f \sum_{i}\left(N T_{r} D_{I S, i}\right)^{2} \\
& =\frac{1}{16 I(I+1)} \frac{2}{2 I+1} f M_{2}^{I S},
\end{aligned}
$$

where $M_{2}$ denotes the van Vleck second moment:

$M_{2}=\frac{4}{15}\left(\frac{\mu_{0}}{4 \pi}\right) \gamma_{I}^{2} \gamma_{S}^{2} \hbar^{2} I(I+1) \sum_{i} r_{I S}^{-6}$.

In order to study the effects of the spin order and the geometry in multispin systems, SIMPSON calculations were carried out for a three-spin $\mathrm{SI}_{2}\left({ }^{27} \mathrm{Al}-{ }^{31} \mathrm{P}-{ }^{27} \mathrm{Al}\right)$ system with $D_{I S}=-400 \mathrm{~Hz}$ and different $C_{Q}$ values $(0.5,2$ and $6 \mathrm{MHz})$ for $I$ spins. The angles between the two dipolar vectors were also varied as $0^{\circ}, 30^{\circ}, 60^{\circ}$, and $90^{\circ}$. The corresponding $f$ values estimated by fitting the initial

\section{Table 1}

Estimated $f$ values for ${ }^{27} \mathrm{Al}-{ }^{31} \mathrm{P}$ and ${ }^{27} \mathrm{Al}-{ }^{31} \mathrm{P}-{ }^{27} \mathrm{Al}$ systems obtained by fitting the

\begin{tabular}{|c|c|c|c|c|c|}
\hline \multirow[t]{3}{*}{$C_{Q}(\mathrm{MHz})$} & \multicolumn{5}{|l|}{$F$} \\
\hline & \multirow[t]{2}{*}{${ }^{27} \mathrm{Al}-{ }^{31} \mathrm{P}$} & \multicolumn{4}{|c|}{${ }^{27} \mathrm{Al}-{ }^{31} \mathrm{P}-{ }^{27} \mathrm{Al}$} \\
\hline & & $\Delta \beta^{D}=0^{\circ}$ & $\Delta \beta^{D}=30^{\circ}$ & $\Delta \beta^{D}=60^{\circ}$ & $\Delta \beta^{D}=90^{\circ}$ \\
\hline 0.5 & 0.9946 & 0.9840 & 0.9617 & 0.9724 & 1.008 \\
\hline 2.0 & 0.8926 & 0.8832 & 0.8690 & 0.8659 & 0.8776 \\
\hline 6.0 & 0.7053 & 0.6990 & 0.7417 & 0.7734 & 0.7621 \\
\hline $0.5,2.0$ & $0.9436^{\mathrm{a}}$ & 0.9340 & 0.9197 & 0.9165 & 0.9282 \\
\hline $2.0,6.0$ & $0.7990^{\mathrm{a}}$ & 0.7916 & 0.8346 & 0.8665 & 0.8550 \\
\hline
\end{tabular}
SIMPSON simulated results to Eq. (16).

a The average of two $f$ values for individual $C_{Q}$ value. part of the dephasing curves $\left(\left|N T_{r} D_{I S}\right|<0.3\right)$ to Eq. (18) were summarized in Table 1. Apparently, the obtained $f$ values are rather independent of the geometries and the orders of the spin systems. The $f$ values estimated from the $S I_{2}$ systems are in good agreement with the value obtained for the SI system, even with a $C_{Q}$ value as large as $6.0 \mathrm{MHz}$. In addition, the simulation for a three-spin $\left(S I^{\prime} I^{\prime \prime}\right)$ system was also carried out. The $C_{Q}$ values of the $I^{\prime}$ and $I^{\prime \prime}$ spins were set to 0.5 and $2 \mathrm{MHz}$, respectively. For comparison, the $f$ values for the two-spin systems $\mathrm{SI}^{\prime}$ and $\mathrm{SI}^{\prime \prime}$ were also determined. As illustrated in Table 1 (bottom two entries), the $f$ value obtained for the $S I^{\prime} I^{\prime \prime}$ system is comparable $(<10 \%)$ to the average of the $f$ values obtained for $S I^{\prime}$ and $S I^{\prime \prime}$. The same conclusion is observed when the $C_{Q}$ values of the $I^{\prime}$ and $I^{\prime \prime}$ spins were set to 2 and $6 \mathrm{MHz}$. Thus, the factor $f$ for multiple-site systems can be approximated by the weighted average of the $f$ values obtained for the corresponding two-spin subsystems. Consequently, once the $C_{Q}$ values of quadrupole nuclei for a material under investigation are determined from experimental data, the $f$ value can be estimated from SIMPSON simulations and thus the van Vleck second moment can be extracted by fitting the initial dephasing data using central-transition SPI-R ${ }^{3}$ to Eq. (18) with the predetermined $f$ value.

\subsection{Measurements on model compounds}

To validate the aforementioned recoupling scheme experimentally, measurements were carried out for two crystalline model samples, namely $\mathrm{AlPO}_{4}-5$ and $\mathrm{AlPO}_{4}-11$. Both model compounds are microporous aluminophosphates whose structures consist of alternating $\mathrm{AlO}_{4}^{-}$and $\mathrm{PO}_{4}^{+}$tetrahedra linked together via bridging oxygen atoms. For the $\mathrm{AlPO}_{4}-5$, which has a single $\mathrm{Al}$ site, the second-order quadrupolar effect (SOQE) was found to be $2.57 \mathrm{MHz}$ based on earlier MQMAS experiments [46]. On the other hand, it has been reported that there are three different $T$ sites with the relative ratio of $2: 2: 1$ in $\mathrm{AlPO}_{4}-11$, corresponding to the $C_{Q}$ values of 2.6, 2.15, and $0.5 \mathrm{MHz}$ and $\eta$ values of $0.45,0.35$, and 0.5 , respectively $[47,48]$. The $f$ values for each $\mathrm{Al}$ site in $\mathrm{AlPO}_{4}-5$ and $\mathrm{AlPO}_{4}-11$ obtained from two-spin SIMPON simulations are summaries in Table 2. In the case of $\mathrm{AlPO}_{4}-11$ which has multiple Al sites, the weighted average of the $f$ values was used for the $M_{2}$ extraction. Under the MAS condition, both dehydrated aluminophosphate samples were found to exhibit a single ${ }^{31} \mathrm{P}$ resonance. Figs. $4 \mathrm{a}$ and $\mathrm{b}$ revealed the experimental signal dephasing using the SPI-R $\mathrm{R}^{3}$ sequence for $\mathrm{AlPO}_{4}-5$ and $\mathrm{AlPO}_{4}-11$, respectively. The experimental data at the initial regime, i.e. $\Delta S /$ $S_{0}<0.2$, were fitted by the Eq. (18) to derive the $M_{2}$. As can be found in Table 2, the $M_{2}$ data deduced from this approach are in good agreements (within ca. 5\%) with those calculated based on the crystalline structures reported in literatures [49,50].

Table 2

\begin{tabular}{|c|c|c|c|c|c|c|}
\hline & \multirow[t]{2}{*}{$\begin{array}{l}\mathrm{C}_{Q} \\
(\mathrm{MHz})\end{array}$} & \multirow[t]{2}{*}{$\begin{array}{l}\text { Site fraction } \\
(\%)\end{array}$} & \multicolumn{2}{|l|}{$f$} & \multicolumn{2}{|c|}{$\begin{array}{l}M_{2} \\
\left(\times 10^{7} \mathrm{~s}^{-2}\right)\end{array}$} \\
\hline & & & $\begin{array}{l}\text { Individual } \\
\text { site }\end{array}$ & $\begin{array}{l}\text { Multiple } \\
\text { sites }\end{array}$ & Calc $^{a}$ & Expt \\
\hline $\mathrm{AlPO}_{4}-5$ & $2.57^{\mathrm{b}}$ & 100 & 0.8934 & - & 7.04 & 7.28 \\
\hline \multirow{3}{*}{$\begin{array}{l}\mathrm{AlPO}_{4-}^{-} \\
11\end{array}$} & 0.50 & 20 & 0.9857 & $0.9029^{c}$ & 7.95 & 8.22 \\
\hline & 2.15 & 40 & 0.8797 & & & \\
\hline & 2.60 & 40 & 0.8846 & & & \\
\hline
\end{tabular}

Summary of related parameters for $\mathrm{AlPO}_{4}-5$ and $\mathrm{AlPO}_{4}-11$ model samples.

\footnotetext{
a Calculated based on the structures reported in literatures [49,50]

b The SOQE estimated by earlier MQMAS experiments [46].

${ }^{c}$ The weighted average of the $f$ value of each individual site.
} 
a

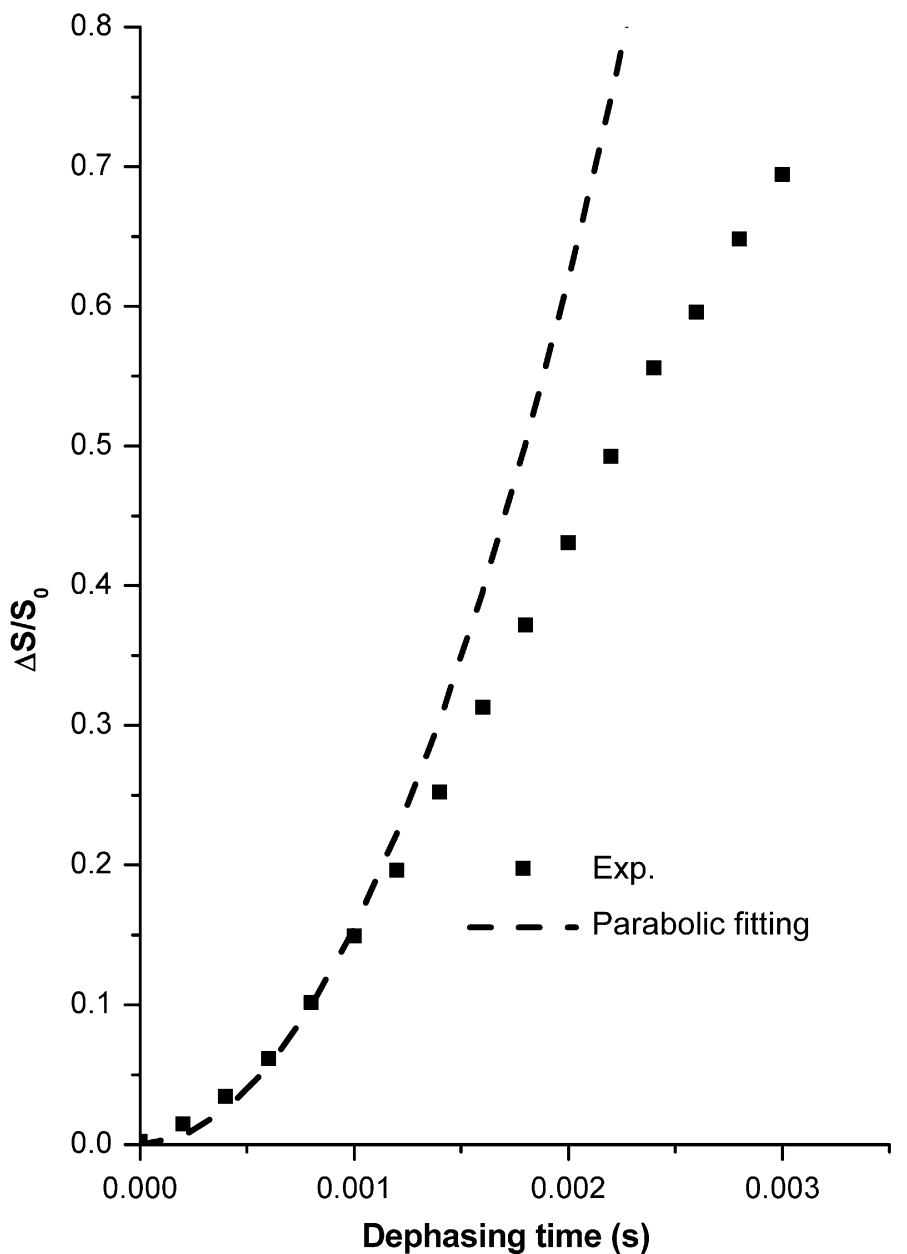

b

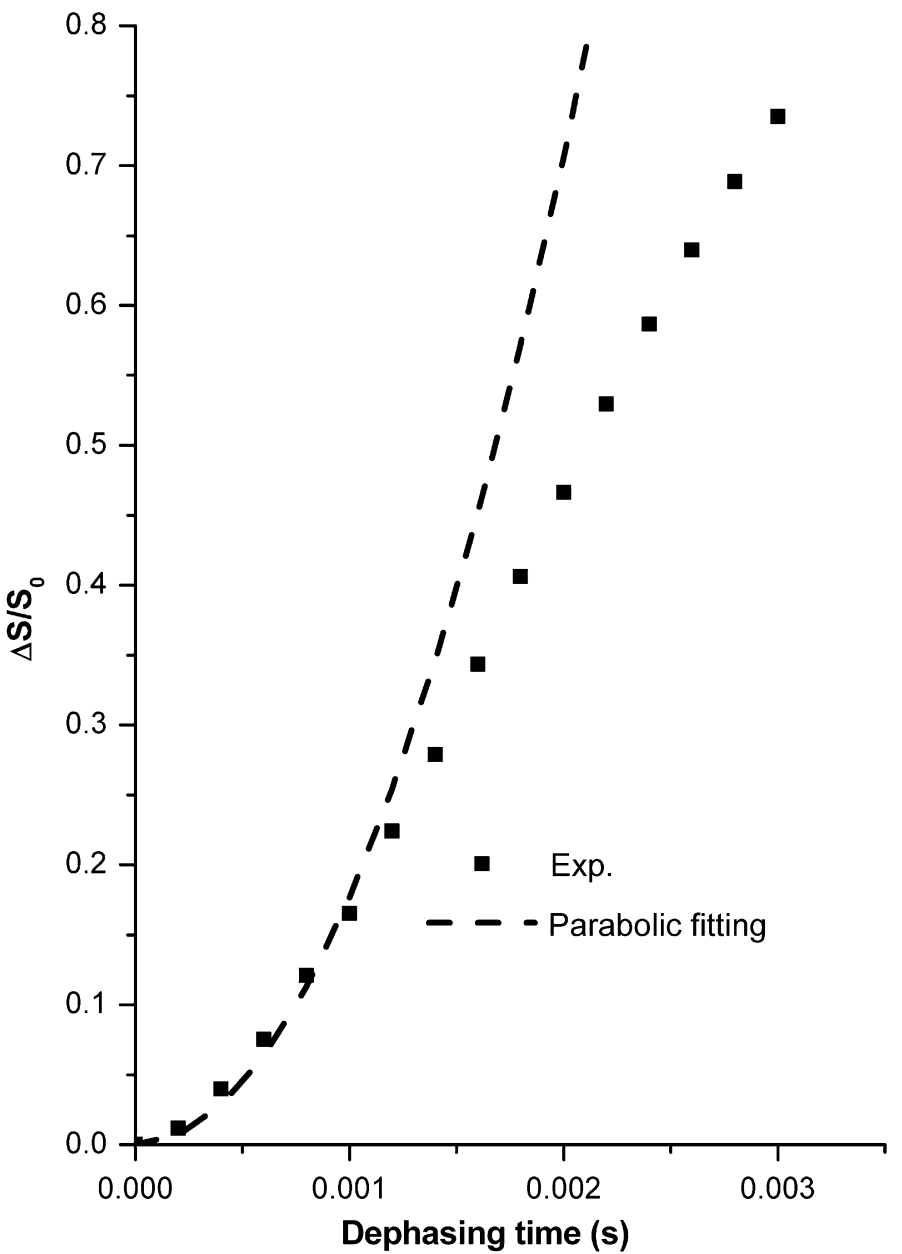

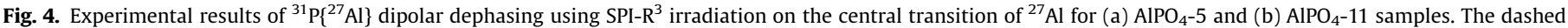
curves represent the parabolic fittings at the initial stage of the dephasing $\left(\Delta S / S_{0}<0.2\right)$.

\section{Conclusions}

We have demonstrated that the dephasing behavior arising from SPI- $\mathrm{R}^{3}$ at the initial evolution stage could be approximated by a parabolic equation modified with a factor $f$, which reflects the influences of the $C_{Q}$. The factor $f$ determined by numerical simulations is found to be independent of the geometry and the order of the spin system. This semi-empirical approach is also validated on systems with multiple sites. The van Vleck second moments determined for $\mathrm{AlPO}_{4}-5$ and $\mathrm{AlPO}_{4}-11$ are in good agreement with the literature data. While the technique such as TRAPDOR and REAPDOR can be applied in the adiabatic regime (slow spinning or strong rf field), our approach will find a general application in the sudden regime (fast spinning and/or weak rf field).

\section{Supplementary materials}

Representative ${ }^{31} \mathrm{P}$ spectra of $\mathrm{AlPO}_{4}-5$ and $\mathrm{AlPO}_{4}-11$ at different dephasing times with and without SPI- $\mathrm{R}^{3}$ recoupling.

\section{Acknowledgments}

The authors are grateful to Prof. Jean-Paul Amoureux at Université des Sciences et Technologies de Lille for helpful discussions. The generous supply of crystalline model compounds from Prof. Soofin Cheng (National Taiwan University) is also acknowledged.

\section{Appendix A. Supplementary material}

Supplementary data associated with this article can be found in the online version at doi:10.1016/j.ssnmr.2009.07.002.

\section{References}

[1] R. Tycko, G. Dabbagh, Chem. Phys. Lett. 173 (1990) 461-465.

[2] T. Gullion, J. Schaefer, J. Magn. Reson. 81 (1989) 196-200.

[3] T.G. Oas, R.G. Griffin, M.H. Levitt, J. Chem. Phys. 89 (1988) 692-695.

[4] T. Gullion, S. Vega, Chem. Phys. Lett. 194 (1992) 423-428.

[5] A.E. Bennett, J.H. Ok, R.G. Griffin, S. Vega, J. Chem. Phys. 96 (1992) 8624-8627.

[6] N.C. Nielsen, H. Bildsoe, H.J. Jakobsen, M.H. Levitt, J. Chem. Phys. 101 (1994) $1805-1812$.

[7] Y.K. Lee, N.D. Kurur, M. Helmle, O.G. Johannessen, N.C. Nielsen, M.H. Levitt, Chem. Phys. Lett. 242 (1995) 304-309.

[8] S. Dusold, A. Sebald, Ann. Rep. NMR Spectrosc. 41 (2000) 185-264.

[9] C.P. Grey, W.S. Veeman, Chem. Phys. Lett. 192 (1992) 379-385.

[10] C.P. Grey, A.J. Vega, J. Am. Chem. Soc. 117 (1995) 8232-8242.

[11] T. Gullion, Chem. Phys. Lett. 246 (1995) 325-330.

[12] Y. Ba, H.M. Kao, G.P. Grey, L. Chopin, T. Gullion, J. Magn. Reson. 133 (1998) 104-114. 
[13] A. Goldbourt, S. Vega, T. Gullion, A.J. Vega, J. Am. Chem. Soc. 125 (2003) 11194-11195.

[14] M. Baldus, D. Rovnyak, R.G. Griffin, J. Chem. Phys. 112 (2000) 5902-5909.

[15] S. Wi, J.W. Logan, D. Sakellariou, J.D. Walls, A. Pines, J. Chem. Phys. 117 (2002) 7024-7033.

[16] A.J. Painter, M.J. Duer, J. Chem. Phys. 116 (2002) 710-722.

[17] J.P. Amoureux, J. Trebosc, L. Delevoye, O. Lafon, B. Hu, Q. Wang, Solid State Nucl. Magn. 35 (2009) 12-18.

[18] G. Mali, G. Fink, F. Taulelle, J. Chem. Phys. 120 (2004) 2835-2845.

[19] G. Mali, V. Kaucic, J. Magn. Reson. 171 (2004) 48-56.

[20] G. Mali, J. Magn. Reson. 185 (2007) 318-325.

[21] M. Eden, H. Annersten, A. Zazzi, Chem. Phys. Lett. 410 (2005) 24-30.

[22] M. Eden, D. Zhou, J.H. Yu, Chem. Phys. Lett. 431 (2006) 397-403.

[23] B. Hu, J. Trebosc, J.P. Amoureux, J Magn. Reson. 192 (2008) 112-122.

[24] Z.H. Gan, J. Magn. Reson. 183 (2006) 235-241.

[25] Z.H. Gan, J.P. Amoureux, J. Trebosc, Chem. Phys. Lett. 435 (2007) 163-169.

[26] J. Trebosc, B. Hu, J.P. Amoureux, Z. Gan, J. Magn. Reson. 186 (2007) 220-227.

[27] S. Cavadini, A. Abraham, G. Bodenhausen, Chem. Phys. Lett. 445 (2007) 1-5.

[28] A. Brinkmann, A.P.M. Kentgens, J. Am. Chem. Soc. 128 (2006) 14758-14759.

[29] A. Brinkmann, A.P.M. Kentgens, J. Phys. Chem. B 110 (2006) 16089-16101.

[30] P.R. Costa, J.D. Gross, M. Hong, R.G. Griffin, Chem. Phys. Lett. 280 (1997) 95103.

[31] M. Bak, J.T. Rasmussen, N.C. Nielsen, J. Magn. Reson. 147 (2000) 296-330.

[32] M. Bak, N.C. Nielsen, J. Magn. Reson. 125 (1997) 132-139.

[33] L. Frydman, J.S. Harwood, J. Am. Chem. Soc. 117 (1995) 5367-5368.
[34] A. Medek, J.S. Harwood, L. Frydman, J. Am. Chem. Soc. 117 (1995) 1277912787.

[35] J.P. Amoureux, C. Fernandez, L. Frydman, Chem. Phys. Lett. 259 (1996) 347355.

[36] J.P. Amoureux, C. Fernandez, S. Steuernagel, J Magn. Reson. Ser. A 123 (1996) $116-118$.

[37] Z.H. Gan, J. Am. Chem. Soc. 122 (2000) 3242-3243.

[38] K.J. Pike, S.E. Ashbrook, S. Wimperis, Chem. Phys. Lett. 345 (2001) 400-408.

[39] S.E. Ashbrook, S. Wimperis, J. Am. Chem. Soc. 124 (2002) 11602-11603.

[40] S.E. Ashbrook, S. Wimperis, J. Magn. Reson. 156 (2002) 269-281.

[41] W. Stroiek, C.M. Felise, H. Eckert, B. Ewald, R. Kniep, Solid State Nucl. Magn. Reson. 32 (2007) 89-98.

[42] W. Strojek, H. Eckert, Phys. Chem. Chem. Phys. 8 (2006) 2276-2285.

[43] W. Strojek, M. Kalwei, H. Eckert, J. Phys. Chem. B 108 (2004) 7061-7073.

[44] M. Bertmer, H. Eckert, Solid State Nucl. Magn. Reson. 15 (1999) 139-152.

[45] M. Bertmer, L. Zuchner, J.C.C. Chan, H. Eckert, J. Phys. Chem. B 104 (2000) 6541-6553.

[46] J.C.C. Chan, H. Eckert, J. Magn. Reson. 147 (2000) 170-178.

[47] P.J. Barrie, M.E. Smith, J. Klinowski, Chem. Phys. Lett. 180 (1991) 6-12.

[48] M.P.J. Peeters, J.W. Dehaan, L.J.M. Vandeven, J.H.C. Vanhooff, J. Phys. Chem. 97 (1993) 5363-5369.

[49] J.W. Richardson, J.J. Pluth, J.V. Smith, Acta Crystallogr. B 44 (1988) 367-373.

[50] G.J. Klap, H. van Koningsveld, H. Graafsma, A.M.M. Schreurs, Micropor. Mesopor. Mater. 38 (2000) 403-412. 